\title{
Thermodynamics and Entropy in Natural and Artificial Systems
}

\author{
Richard D. Sauerheber \\ STAR Center, Palomar Community College, San Marcos, Califorina \\ RSauerheber@palomar.edu
}

Abstract: This monograph clarifies thermodynamic theories by examining several physical, biological, and chemical systems in nature. The compromise between achieving a maximum entropy, which drives a process forward, but a minimum energy in a system to achieve stability in an equilibrium condition, governs the characteristics of change in nature. Whether it is growth of an organism, the propagation of light and mass through space, the reactivity of chemical processes, or the energy changes in physical processes such as the formation and then dissipation of hurricanes or the freeze-thaw cycles of sea ice, the total entropy or randomness of the system and surroundings must be positive in order to proceed forward. However, systems at equilibrium where no further entropy change occurs are still characterized with much dynamism. And favorable entropy alone does not ensure a particular process will proceed for a variety of reasons, all consistent with the fundamental laws of thermodynamics.

\section{INTRODUCTION}

Thermodynamic principles deem that changes in the entropy or randomness of a system and its surroundings, first introduced by Rudolf Clausius in 1850, determine whether a process is spontaneous and favorable or not. Opening a carbonated beverage spontaneously causes a pop sound because the carbon dioxide trapped gas quickly expands into the atmosphere thereby increasing the entropy of the gas. Entropy is basically the energy content of a system at a particular temperature with units of Joules per Kelvin. This leads to the definition of Gibbs available, or free, energy, where favorable processes are those that produce a negative change in free energy where the sum of the entropy change of the system $\Delta S_{\text {system }}$ and any accompanying entropy change in its surroundings $\Delta S_{\text {surr }}$ produces a total entropy change $\Delta S_{\text {total }}$ that is positive at a particular temperature $T$. Here,

$$
\Delta S_{\text {total }}=\Delta S_{\text {system }}+\Delta S_{\text {surr }}
$$

for any particular physical or chemical change and the delta symbol $\Delta$ refers to the difference in magnitude between final and initial states after any process has taken place. Since entropy changes in the surroundings typically involve exchange of heat and work, $\Delta S_{\text {surr }}=\Delta H / T$ where $\Delta H$ is the enthalpy change $(\Delta E+\Delta[P V])$ in a system, $E$ is the internal energy $P$ is pressure, and $V$ is the volume. Multiplying by -T and rearranging, $\Delta H$ - $T \Delta S_{\text {system }}$ is the formula for free energy in Joules $\Delta G=-T \Delta S_{\text {total }}(1)$ which Josiah Gibbs in 1873 considered as the energy available to perform work. The role played by entropy is examined here for several natural systems. Some of the conclusions reached are very controversial but nevertheless need to be discussed.

\section{Biology}

One commonly discussed example is growth in living organisms. Here chemical randomness and thus entropy is reduced since nutrient molecules are assimilated and used to synthesize macromolecules and cell structures in growth. This does not violate thermodynamic principles because growth in animals is associated with increased production of gaseous carbon dioxide released into the surroundings, plus to some extent release of heat from warm blooded animals with increases in the entropy of the surroundings.

From the second law of thermodynamics we know that any spontaneous change in a system is accompanied by an increase in total entropy (3). However, the extrapolation that any expected positive total entropy change 


\section{Thermodynamics and Entropy in Natural and Artificial Systems}

ensures that a particular process will indeed occur is not correct. Obviously many species have gone extinct in history even though entropy considerations had been favorable for growth. Predators and disease eliminating populations would be two examples. Organism viability is not guaranteed from favorable entropy considerations, even though thermodynamic favorability is indeed required for growth.

From a biochemistry perspective, for energetically unfavorable reactions such as dehydration reactions in general anabolism, the concurrent increase in entropy due to the conversion of adenosine triphosphate ATP into adenosine diphosphate ADP and phosphate causes such reactions to proceed spontaneously. The positive total entropy change that must occur is partly due to increased randomization of matter into products.

The growth of plants occurs by the capture of atmospheric carbon dioxide and its conversion to phosphoglyceric acid in photosynthesis $(4,5)$. Gas capture such as this represents a huge decrease in entropy. The process is favorable however for many reasons. The plant releases oxygen gas in equal molar amounts into the atmosphere, and the metabolic reactions in the Calvin-Benson cycle (1950) utilize ATP which hydrolyses to release energy and form ADP and phosphate. The overall process has a free energy that is negative since the total entropy change is positive.

The free energy available to perform work is readily visible during the contraction of skeletal muscle. The actin and myosin filaments contract to shorten the fiber with an entropy decrease. But this is accompanied with ATP hydrolysis with its entropy increase and release of chemical bond energy. The process is then spontaneous with a $\Delta S_{\text {total }}$ that is positive with a negative energy $\Delta \mathrm{G}$, which is the work $w$ of filament contraction due to mechanical oscillations of the myosin cross bridges that repeatedly attach and disengage actin binding sites to pull the filaments along myosin to shorten the fiber. Some of the released energy emerges as heat and the rest is free energy available to perform this work.

Biologic cell surface membranes are systems with intermediate entropy content. This is because the lipid bilayer matrix is composed of phospholipids with fatty acid chains that are highly flexible. The fluidity of various membranes has been examined in detail with fatty acid labels and electron spin resonance spectroscopy (10). The bilayer however also has much structural order as well, where the outer layer contains phospholipids that are not highly negatively charged at extra cellular $\mathrm{pH}$ with a fluidity that is minimized by extracellular millimolar calcium and magnesium, while the more negatively charged inner layer has a fluidity that is minimized by millimolar cytoplasmic magnesium inside the cell where calcium is negligible. Entropy dictates that proteins in the fluid bilayer rotate and undergo lateral diffusion and other motions in a random orientation to allow proper function.

At physiologic temperature in mammals, the membrane bilayer is above any phase transition temperature and is in a relatively fluid state except for domains in which cholesterol is embedded. Cholesterol decreases the fluidity and thus the entropy of the membrane bilayer. The most rigid membranes found are the intestinal brush border and red cell membranes having high cholesterol to phospholipid ratios around 1:1. Peripheral tissue membranes such as fat, liver, and muscle are more fluid with a much lower cholesterol to phospholipid ratio $(20,21)$.

Birds and mammals are homeothermic organisms, where life is only possible when the internal temperature is regulated at a fixed value, humans around $98.6^{\circ} \mathrm{F}$ and other animals ranging from $96-103^{\circ} \mathrm{F}$. All other organisms are poikilothermic and function at the temperature of the environment in which they live, over wide ranges.

It must be noted that the notion that warm-blooded animals such as birds somehow descended directly over time from ancestors that were cold-blooded, such as dinosaurs, is not supported by thermodynamic considerations. 


\section{Thermodynamics and Entropy in Natural and Artificial Systems}

There are no homeothermic animals known that live fixed at $70^{\circ}, 80^{\circ}$, or $90^{\circ}$, etc. temperature set points. Genetic alterations confer tremendous variability within a genus of life so that many subspecies in form exist within it, but changes that are substantial enough to cause non-breeding with a parental strain are typically lethal. That microevolution within a genus is proven and observed, but macroevolution between genera has not been proven or observed, has been described in more detail elsewhere (16).

Dinosaurs and other living poikilotherms do not release heat to the surroundings compared to metabolic heat radiation in homeotherms. This demonstrates that gas release, rather than metabolic heat loss, is mostly responsible for the total entropy increase necessary for living organisms to exist.

\section{Chemistry}

For simpler systems such as the dissolution of salts in aqueous solution, increased randomness and entropy of the system after dissociation into free ions drives the process forward. However a solution saturated with a particular salt cannot allow further dissociation of that salt, in the common ion effect. As with biological species that do not grow in spite of favorable entropy, the additional dissolution of salt does not occur in spite of expected increases in entropy. This is not due to any decrease in entropy of the surrounding solution molecules. Rather, entropy increases expected in the system by adding more scattered salt ions must be countered by other factors.

One possibility is that electrostatic repulsion of concentrated like ions blocks further dissociation without involving an entropy decrease in the surroundings. In this way, additional solid salt simply remains solid. Since the actual entropy change would be zero, this is consistent with the principal that positive total entropy is necessary for a process to change the net composition of the system. The electric potential energy increase for the system, if additional ions could be incorporated, would offset any increase in system entropy so that $\Delta \mathrm{S}_{\text {total }}$ is zero, the saturated system is at equilibrium, and no additional change in concentration takes place.

Although directed processes which are favorable to occur always have total entropy changes that are positive, the value does not correlate with reaction speed. Dynamite exploding is a rapid reaction to form products with great entropy, but the degeneration of voltage in a battery is also highly favorable but is a relatively slow process for a given amount of matter. Reactions with favorable entropy do not proceed unless the energy of the reactants is sufficient to activate the process. Lighting paper with a match provides the activation energy for the heated molecules to break bonds which then allows the entropy favorable process to continue without the match, and at a rate that is determined by other factors.

\section{Entropy and Equilibrium States}

The salt solution example above demonstrates the role of entropy in achieving the equilibrium condition. A system is at equilibrium when it has no further tendency to change its average properties. Thus the entropy of a system can increase until no further spontaneous changes can occur. When the entropy reaches a maximum, the energy in a system has dispersed and the system no longer changes and equilibrium has been attained (3). The drift of a system toward equilibrium in nature is governed by two factors. One is the tendency toward minimum energy, and the other is the tendency toward maximum entropy and chaos. In the saturated salt solution above, energy minimization and entropy maximization strike a compromise. Dissolving more ions would increase to a larger entropy but would also not allow a minimum energy. Precipitating ions from solution would minimize electrical potential energy but would not allow the maximum entropy. The opposing factors balance at equilibrium (Figure 1). 


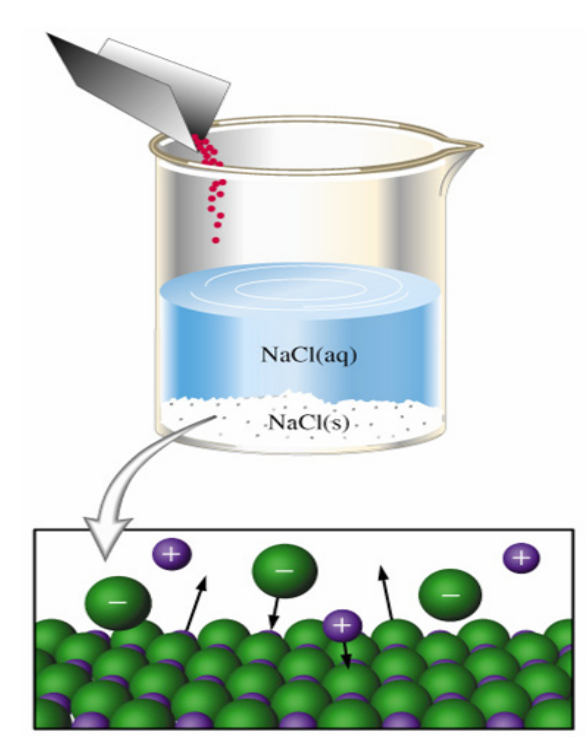

Fig1. The salt solution is saturated so that sodium cations and chloride anions dissolve and precipitate at equal rates. This ionic exchange is a dynamic process but the system is in static equilibrium since no net mass is transferred from one location to another.

In reality, although the saturated salt solution at equilibrium no longer changes the concentration of ions in solution, nevertheless ions dissolve from the solid and ions also precipitate from the liquid continuously at equal rates. These interchanges occur with no change in total entropy. Most systems at equilibrium contain ongoing processes such as this.

The means by which a system reaches a static equilibrium were first described from original experiments with gas conducted by James Joule in 1852 (3). For an isolated system of gas molecules, without exchange of matter or heat with the surroundings, or change in temperature or thus energy of the gas, molecules diffuse down a concentration and pressure gradient with net transport or flux until the pressure is the same throughout the system. Then a static equilibrium is reached where entropy becomes a maximum (Figure 2).

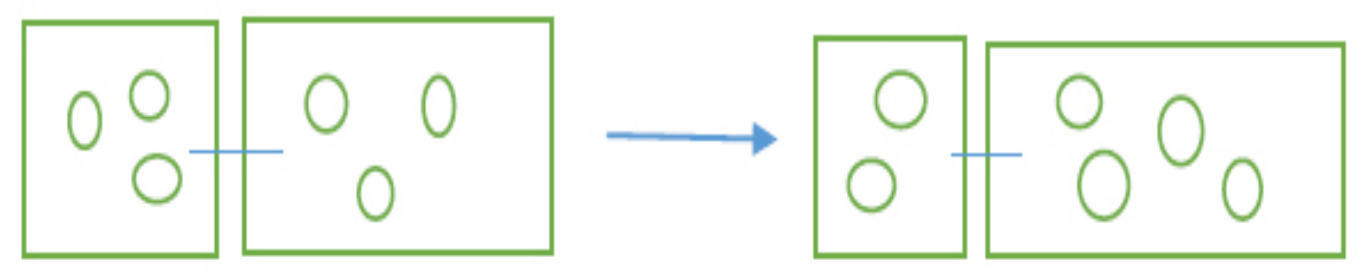

Fig2. Two containers of gas samples represented as containing equal moles, but one at twice the pressure and half the volume as the other so that PV is constant. The energy content of both samples is the same PV $=n R T$. Since heat exchange with the surroundings is insulated. Notice that initially both containers had the same number of gas molecules but afterward the twice larger volume has two times as many molecules than the smaller volume. The shift is entirely due to a randomness form of entropy where mass has been dispersed. Originally the container on the left had a higher energy concentration than for the container on the right so E/Vleft $=2 E / V r i g h t$. This type of expansion is named after James Prescott Joule who conducted a similar experiment in 1845 after similar results were obtained by John Leslie in the early 19th century and Joseph-Louis Gay-Lussac in 1807. 


\section{Thermodynamics and Entropy in Natural and Artificial Systems}

\section{Static and Dynamic Equilibrium}

Systems in static equilibria contain materials at fixed concentrations without net mass exchange, like a closed container of water or gas. Here molecules are in a dynamic state of vibration, rotation, and translational motion. In solid states, atoms contain electrons that oscillate at rapid rates. But there is no net, directed transfer of mass or energy dissipation so $\Delta S=0$. In matter, electrons travel so rapidly that an object visually appears as though electrons are motionless.

Chemicals naturally follow laws of thermodynamics to attain equilibrium. For example, all reactions are characterized by an equilibrium constant $K$ such that products divided by reactants, raised to their coefficients as exponents, is a fixed value at any particular temperature. For dynamite exploding, the $K$ is huge since the reactant virtually disappears into product gases. Many organic reactions typically have $K=1$ since products and reactants have comparable equilibrium molarities, and can be labeled reversible. For the production of aspirin $\mathrm{P}$ from acetic anhydride $\mathrm{A}$ and salicylic acid $\mathrm{B}$, an equilibrium mixture is characterized by

$$
K=[\mathrm{P}] /\{[\mathrm{A}][\mathrm{B}]\}=1
$$

To increase yield, simply increase the molarity of one or both reactants. The chemicals will automatically adjust themselves to re-attain the equilibrium ratio $K$. Initially if all species were at 1 molar, $1 /\{(1)(1)\}=K$ $=1$. Doubling the A reactant, after equilibium is re-attainted, $K=(1+x) /\{(2-x))(1-x)\}=1$, and $\mathrm{x}=0.268$ so the product aspirin concentration is now $1.268 \mathrm{M}$. Here $[\mathrm{A}]=1.73 \mathrm{M}$ and $[\mathrm{B}]=0.732 \mathrm{M}$. The disturbed system proceeds in the forward direction to equilibrium because conditions were changed so that $\Delta S_{\text {total }}>0$. Any such physical or chemical process under a given set of conditions where this is so cannot follow the reverse direction because entropy demands it proceed spontaneously forward.

A system in dynamic equilibrium is one in which the concentration of materials does not change over time, and yet materials are interchanging, such as a pool filled with water that is being drained and filled at the same rate. The water concentration remains the same while molecules are replaced on a net basis in this dynamic state. Similarly, the constancy of the blood glucose concentration is a state of dynamic equilibrium where ingested food occurs concomitant with tissue metabolism, and water intake matches urine production so that glucose and water concentrations remain static over time.

Natural processes that proceed spontaneously in one direction are generally not strictly reversible since equilibrium conditions are not maintained during the process (3). But since chemical reactions can be reversible if conditions are changed so that $\Delta S_{\text {total }}>0$ again, and many natural systems involve chemical change, some could be labeled essentially reversible. For example sea ice that melts in summer when ambient temperature $T$ is above that of the ice $T_{\text {ice, }}$, and freezes in winter when $T<T_{\text {ice, }}$, where in both cases $\Delta S_{\text {total }}>0$.

In both dynamic and static equilibria therefore, motional activity is rampant. In solid salt solutions even well below $0^{\circ} \mathrm{C}$ for example, electrons from a current source travel through the solid much like electrons on a metal wire and both systems increase in conductivity as the temperature is lowered (9). Although electron concentrations are constant as current passes through the system, electrons enter and leave at equal rates in an equilibrium dynamic steady state where mass is transferred over space with time. Figure 3 describes current in a bulb wire as a dynamic equilibrium system. It must be that the total entropy change $\Delta S_{\text {total }}$ in dynamic equilibrium systems, where mass or energy are transported on a net, directional basis, is positive to

be spontaneous. And in static equililbria without net transport of energy or mass through a region, the $\Delta S_{\text {total }}$ associated with the dynamic processes present is zero. 


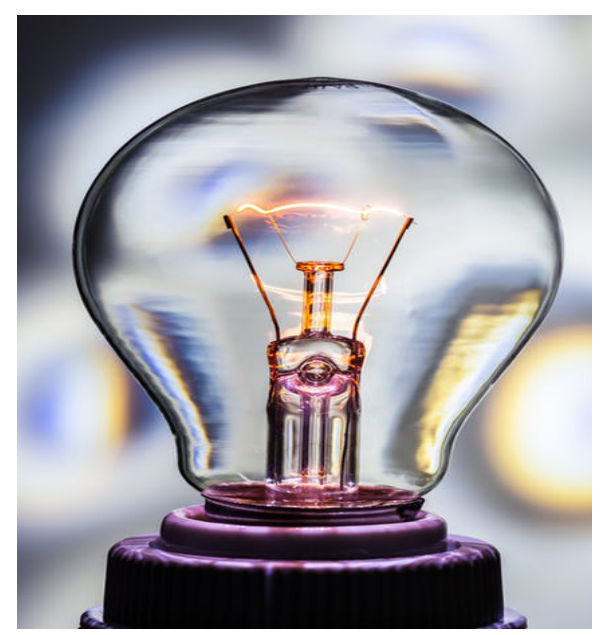

Fig3. Static and dynamic equilibrium systems coexist in an electric light bulb. Electrons slowly flow through filament wires in an electric current to emit light due to changes in energy levels during travel. Since mass is transported on a net basis from one place to another while electron concentrations in the wire remain fixed at any time, the wire is a dynamic equilibrium system. The gas enclosed in the bulb after equilibration to a fixed temperature contains constant energy. Gas molecules randomly diffuse throughout the bulb at a fixed concentration so the enclosed mass is in a state of static equilibrium. (Massless light photons travel through the gas at constant intensity and are not considered part of the gas system for simplicity). Heat transfer from the wire through the gas into the atmosphere is a dynamic equilibrium process.

\section{Physiology}

Glucose uptake into cells from extracellular fluid with a higher concentration continues much like the Joule experiment where gasses flux toward static equilibrium. Except in this case the entropy driven process does not end, because inside the cell glucose is absent, being quickly converted to glucose-6-phosphate. In this way, a concentration gradient is always present so that entropy randomization causes glucose uptake to feed the cell without requiring energy expenditure for it, distinct from the energy required for metabolism of the sugar after entry (Figure 4).

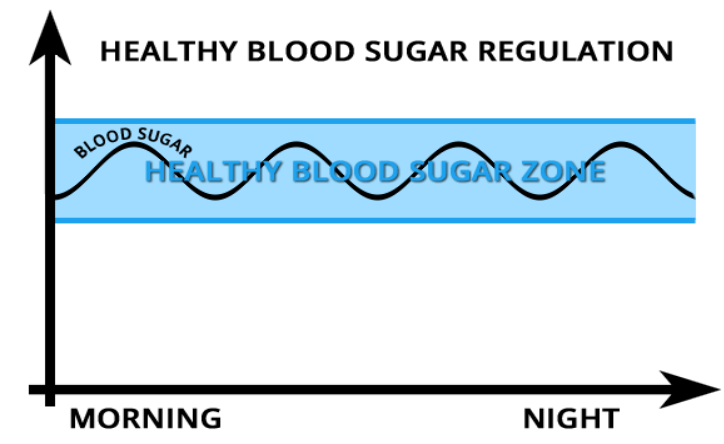

Fig4. Blood glucose levels remain relatively constant because of ingested foodstuffs during mealtime or release of glucose from liver glycogen between meals, coupled with glucose uptake and metabolism by tissues to produce ATP and carbon dioxide. Whole body homeostasis refers to a dynamic equilibrium state since mass is transported on a net basis from intestines to peripheral tissues. Early morning glucose elevations are caused by cortisol released form adrenal glands to prevent hypoglycemia before eating. Breakfast, lunch and dinner meals are followed with elevations that are re-normalized by insulin. Glucagon and other hormones prevent fasting hypoglycemia as well. Although the energy and entropy content of the blood are relatively fixed, the process is thermodynamically favorable due to increased entropy from net mass transfer and the metabolic production of gas. 


\section{Thermodynamics and Entropy in Natural and Artificial Systems}

Detailed experimental work over the past many decades has helped to elucidate the mechanism by which the pancreatic hormones insulin and glucagon regulate blood glucose levels throughout the day. During meal eating, insulin secretion into the blood stimulates membrane glucose transport protein to increase glucose uptake rates in fat and muscle tissue about two-fold over basal rates in the absence of insulin between meals. Glucagon prevents hypoglycemia during periods of fasting by increasing the activity of membrane adenylate cyclase which stimulates glycogen phosphorylase to release glucose into the bloodstream from intracellular glycogen stores in muscle and fat. In this way homoeostasis is maintained where glucose level swings are not excessive as a function of time during and between meals.

Glucose transport proteins intrinsic in the membrane are activated, without unnecessary structural changes in the hydrophobic bilayer (11), which would require unnecessary expenditure of energy. This is consistent with the fact that stimulation by insulin during meals, as well as the return to basal glucose uptake rates after meal eating,are very rapid. Insulin fully stimulates glucose uptake rates at physiologic temperature within two minutes (12). Moreover, drastic changes in the fluidity of the membrane do not affect this time-dependence.

It must be noted that disturbances in the normal homeostatic mechanisms that maintain glucose levels commonly occur in type II diabetes where caloric intake exceeds the ability of tissues to uptake glucose in response to the elevated fed state levels of insulin. Insulin is the only hormone, which is opposed by 14 other hormones, that promotes storage of glucose, fat, and protein, and also directly inhibits glycolysis, lipolysis, and proteolysis.

Type II diabetes is thus not an actual disease but instead is a metabolic condition where dynamic steady state glucose levels are not being maintained, due to excessive loading. Henry Le Chatelier's principle (1898) dictates that for equilibria that have been perturbed by excess reactants, this decreased entropy causes a shift toward products to re-achieve maximum entropy toward equilibrium again. But this can only occur when excess reactant loading is stopped. Indeed, the type II condition (but not Type I diabetes which is an insulin deficiency disease) is readily corrected by a brief fast followed with iso-caloric eating as long as any glucose-lowering drugs are first discontinued to prevent hypoglycemia (13).

A system which physiologists generally regard as being in a dynamic steady state is actually quite complex, namely the Ernest Starling hypothesis (1896) for nutrient and waste exchange in blood capillaries. Oxygen gas, glucose, and other small-sized nutrients along with fluid from blood plasma enter extracellular fluid in peripheral tissues from the arterial side of a capillary. On the venous side carbon dioxide, carbonic acid, bicarbonate, and other waste products such as nitrogenous metabolites, and fluid enter the capillary to return to the heart and lungs. This represents a dynamic steady state system but it is much different than the above examples since the molecules that leave the blood are distinct from the substances that enter later.

Both effusion and influx are caused by entropy increases for molecules moving down concentration gradients, but this is aided on the arterial side by the slightly higher blood hydrostatic pressure from the pumping action of the heart. On the venous side the flux is aided by the higher osmotic pressure in the blood due to the presence of the large proteins namely albumin (Figure 5). In this way gas exchange can occur with the atmosphere at the lungs, and metabolic waste substances are eliminated by the kidneys into the urine. Although still regarded technically as a not fully proven hypothesis, the system thoroughly explains the expected and measured differences in $p \mathrm{H}, p 02, p \mathrm{CO}$, and the levels of glucose and other metabolites between arterial and venous blood (where $p=-\log$ of species concentration). 


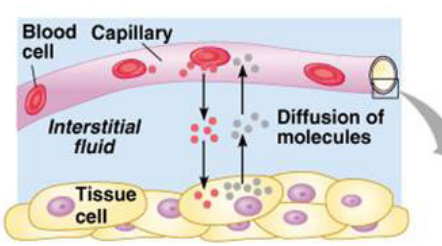

(a)

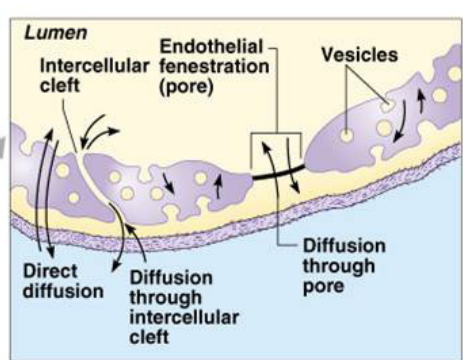

(b)

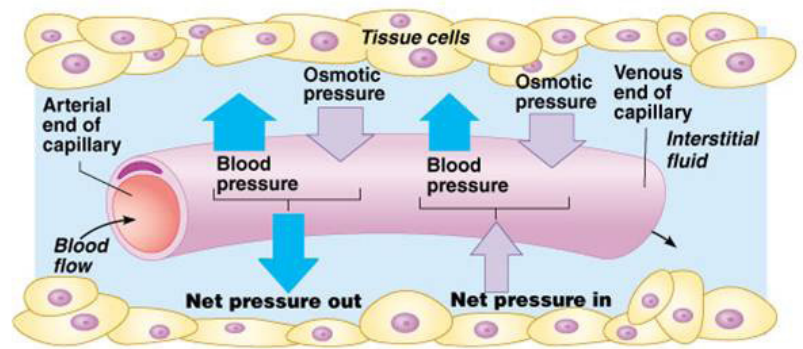

(c)

Fig5. Diagram of the Starling hypothesis of capillary exchange. At the arterial end where blood hydrostatic pressure exceeds osmotic pressure, nutrient molecules, oxygen, and fluid efflux from the blood into extracellular space of tissues. At the venous end where osmotic pressure exceeds blood hydrostatic pressure, fluid, carbon dioxide and other wastes diffuse into the vessel from tissue space.

Returning to gases released from metabolism in living organisms, the atmosphere is in a state of dynamic equilibrium where carbon dioxide enters the atmosphere and oxygen is removed during animal respiration of carbohydrates, but oxygen is replenished and carbon dioxide is removed during plant photosynthesis (Figure 6). Thus the average content of carbon dioxide and oxygen are maintained approximately constant over time, while the gaseous matter is shuttled from one place to another on a net basis. Here the entropy change is approximately zero for the atmosphere as a system, but the process continues since the entropy changes for the plants and animals are both positive. This would be stopped when the sun runs out of light, or if all plants were somehow destroyed, etc. to end the mass/energy exchange.
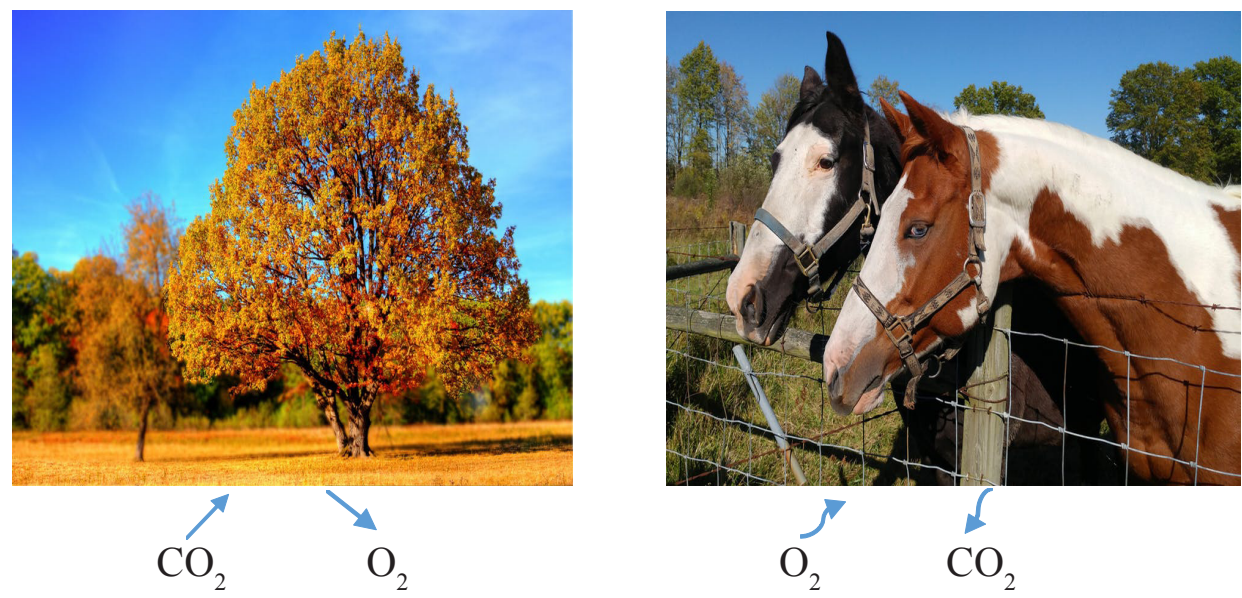

Fig6. Atmospheric dynamic equilibrium. Animals exhale carbon dioxide and inhale oxygen gas for respiration of foodstuffs. Plants incorporate carbon dioxide (day or night) and release oxygen gas (daytime only), so net mass transfer from place to place occurs while the total concentrations in the atmosphere remain relatively constant. 


\section{Thermodynamics and Entropy in Natural and Artificial Systems}

\section{Plant Leaf Biology}

One long-standing vexing problem is how leaves on plants turn so that surfaces face the direction of greatest light intensity. This also is an entropy supported phenomenon. Oxygen gas release from plants is greatest in tissues undergoing the most rapid rate of photosynthesis. Much like poking a hole in a balloon to allow gas to escape, the increased metabolism and release of gas from the leaf portion facing the sun is a huge increase in entropy while the light dependent reaction is in full operation. Less exposed portions turn to allow release of more gas (Figure 7A), somewhat like a balloon that flies left when the punctured hole is on the right. The release of oxygen gas from water entering the plant leaf from the roots represents a highly favorable dynamic equilibrium system.

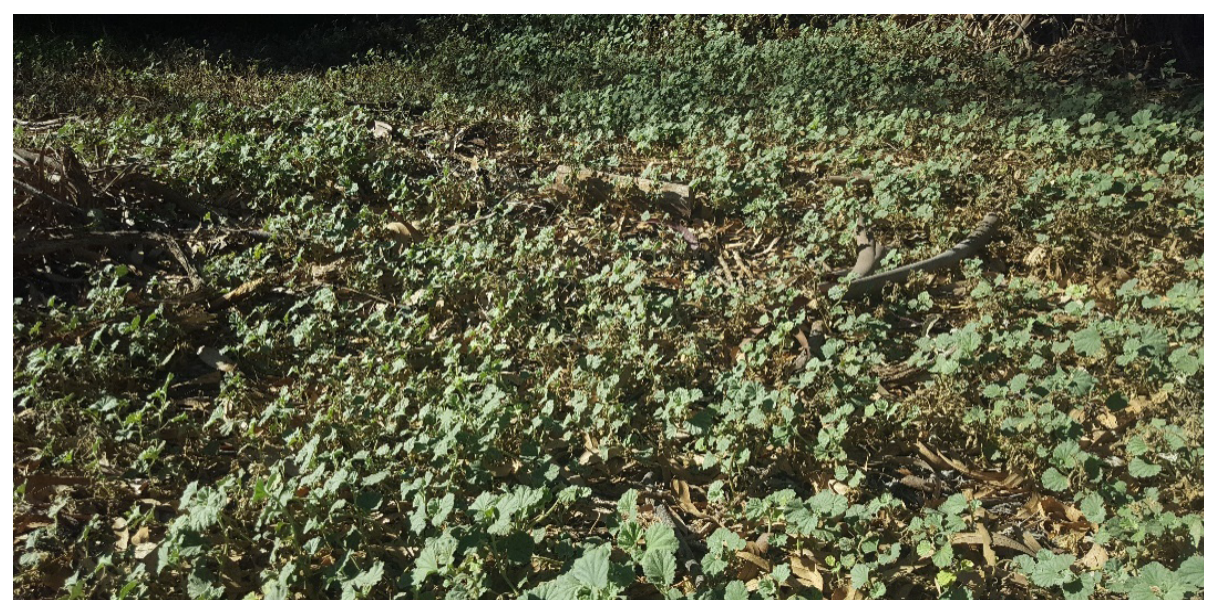

Fig7A. Every leaf in the entire field of young plants faces the direction of the sun to the left. Each day after sunrise, electrical activity in the exposed leaves begins. Light absorption by chlorophyll causes excitation of electrons into cytochromes and replenishment of electrons from water to form hydrogen ions and oxygen gas released into the atmosphere in a dynamic equilibrium process with great increase in entropy.

It has been published that deciduous trees lose leaves in autumn due to sunlight limitations, rather than cooler temperatures. Temperate coastal San Diego County, California in 2018 provided an environment in which this could be tested since ambient temperatures did not decline substantially from late August to early January this particular year. Leaves were examined on apple, plum, apricot, and tangerine trees grown in a small area where watering, sunlight, soil, and environmental weather conditions were the same. Here total sun irradiance averages $5.2 \mathrm{kwatts} / \mathrm{m}^{2}$ per day in mid-summer and $4.3 \mathrm{kwatts} / \mathrm{m}^{2}$ per day in mid-winter (22). This is a consistent variation independent of weather and atmospherics, being caused by the regular yearly orbit of the earth around the sun. Irradiance begins to decline significantly in September (12 hours daylight per day, compared to 14 hours daylight per day in June) where leaf senescence in all tree species began. This causes ATP levels generated from chloroplast photosystems to decrease and is associated with degeneration of chloroplasts. Cell ATP levels are then partially maintained by respiration as the leaves turn color. The increased entropy normally caused by oxygen gas evolution and ATP splitting in chloroplasts is reduced when sunlight intensity declines, so the process is driven by a decreasing positive system entropy due to gas evolution. Eventually ATP hydrolysis falls sufficiently to kill the leaf cells. This is accompanied with intracellular alterations that cause lysosomal enzymes to begin to degrade tissue components into smaller higher entropy fragments and to destroy the leaf which also then is a spontaneous but different process. The leaf material then falls from the tree due to gravity which is another spontaneous process and eventually $\Delta$ Ssystem $=\Delta$ Stotal $=0$, but of course the degraded materials could be re-used as substrates for another living system subsequently.Apple and plum tree leaves in 2011-18 were found to fully shed all leaves later in winter (January, 2018) than apricot tree leaves (December, 2017). The tangerine tree with a lemon branch graft retained all leaves throughout the year, like evergreen trees, in spite of reduced sunlight exposure (Figure 7B). 

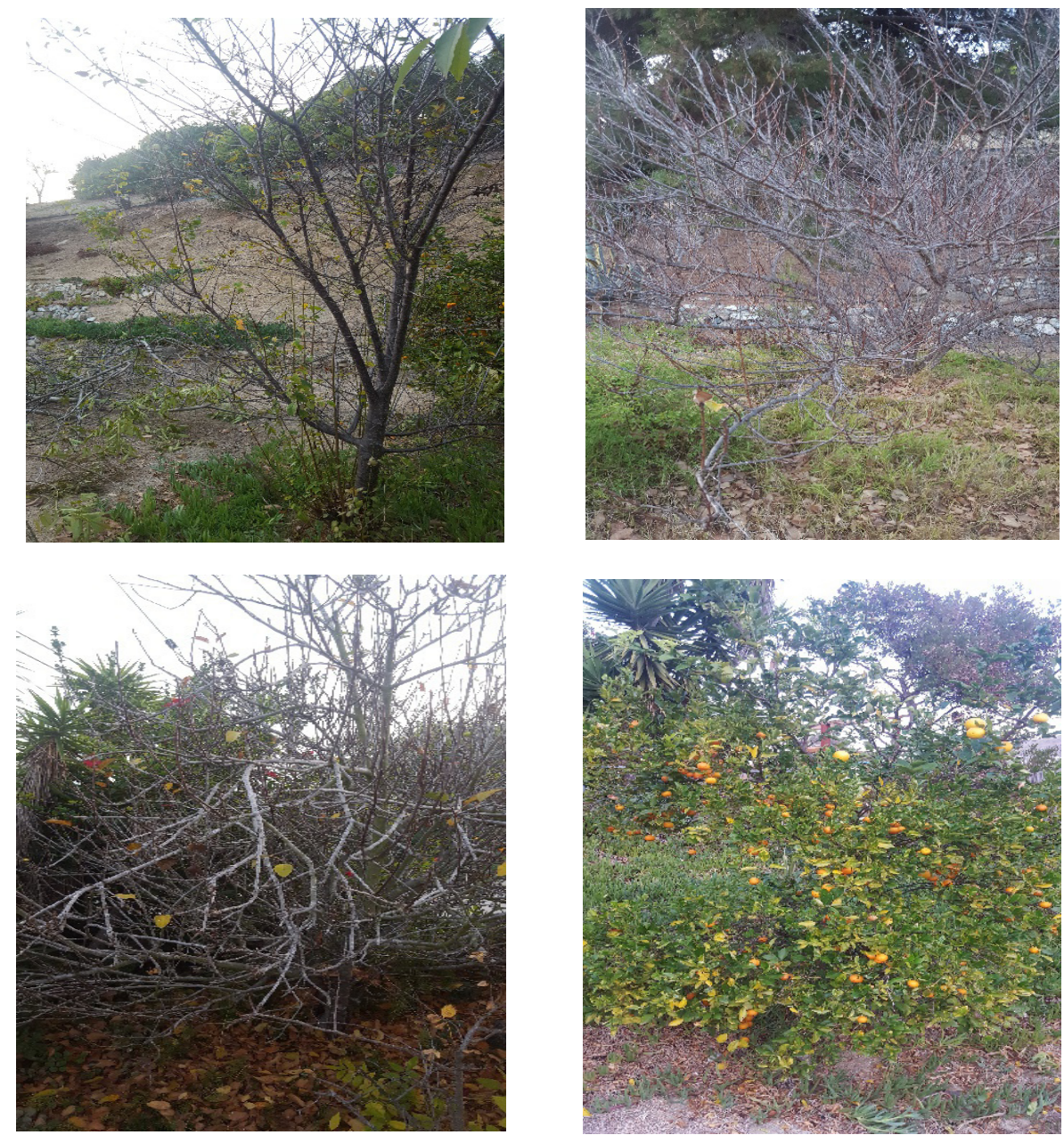

Fig7B. The apricot tree has lost all leaves but one by January 4, 2018 (top right).The apple tree had a dozen remaining leaves (lower left), and the plum tree had several dozen remaining at this date (upper left). A citrus tangerine tree with a grafted lemon branch is evergreen (lower right). Ambient temperature remained mild between $70^{\circ} \mathrm{F}$ daytime and $55^{\circ} \mathrm{F}$ at night, not significantly different than late summer.

These observations indicate that the genetic makeup of trees also modulates the leaf senescence response to decreased sunlight since the various species of tree were found to lose leaves at different rates. Citrus trees are genetically designed to retain green leaves throughout winter. Earlier studies (24) correlated senescence,in 1390 species of deciduous trees, with leaf color change and leaf drop. Some plum tree varietieswere half-senesced at day 268, while this occurs in some magnolia tree varieties at day 300. Results in San Diego are consistent with such differences between species. In March, new leaf growth begins when sunlight duration is 12 hours, up from winter daylight hours in December at 10 hours per day, but new leaf growth is temperature sensitive as well. Increased irradiance causes increased photosynthesis with ATP production and oxygen gas release from chloroplasts, with ATP hydrolysis and leaf growth, where again $\Delta S_{\text {total }}>0$.

\section{Entropy in Human Diseases}

Human pathologic conditions are sometimes caused by increased entropy and in other cases decreased entropy. The three lethal conditions with highest incidence in the human population are heart attacks, strokes, and pneumonia. Growth of bacteria in bronchioles is normally opposed by extracellular secretions. In cases where 


\section{Thermodynamics and Entropy in Natural and Artificial Systems}

this is minimized, bacteria can grow into spaces in lung tissue, as chemicals and biologic organisms tend to expand in space since entropy increases. Lung tissue infected with a particular Streptococcus species that is endogenous in the bronchi is the most common cause of cases of fatal pneumonia. Heart attacks and strokes are caused by insufficient aeration of brain and heart cell tissue so that aerobic respiration is minimized and ATP levels drop. The decreased entropy from lowered ATP hydrolysis when ATP levels below about $0.1 \mathrm{mM}$ is sufficient to be lethal to cells. Death of heart cells is defined as a heart attack and death of brain cells is a stroke. This is because ATP hydrolysis with its increased entropy is normally required to drive the sodium potassium ATP as enzyme transporters intrinsic in the cell surface membrane. Insufficient activity disrupts the normal gradient of sodium outside the cell and potassium inside and blocks normal membrane functions.

\section{Matter Physics}

It is sometimes thought that gravitational, electric, and magnetic fields may cause phenomena that are not explained merely by thermodynamic considerations. Dropping an object toward the earth causes it to be attracted to the ground so that this system appears to exhibit decreased entropy or randomness. In a vacuum there would be no conceivable increase in entropy of the surroundings to allow the action to be thermodynamically favored. Because the fall spontaneously occurs, the fall obviously must not violate thermodynamic truths. But at first glance it appears to occur for reasons other than entropy considerations.

One argument might be that the absence of the object from its original location in the earth's gravitational field increased the entropy of the surroundings sufficiently to drive the process forward. This however is not consistent with known features of gravitational fields, where the presence of any known material in the field does not diminish or attenuate the strength of the field at that location. For example, consider the earth-moon system that oscillates around a common barycenter point. The barycenter smoothly orbits the sun at all times, regardless of whether the earth is positioned between the barycenter and the sun, or the earth and moon are lateral to the barycenter. The gravitational force caused by the sun remains precisely the same at all times, which ensures its smooth elliptic continuous orbit, while the extra gravity from the earth on the moon (and the moon on the earth) causes the two to rotate around each other. Unlike electrical and magnetic fields which can be attenuated with dielectrics and various substances, gravity therefore is an unrestrained, irreducible field.

Another argument is that the collision of an object with the earth causes increased entropy of the molecules involved and thus the total entropy of the system plus surroundings increases to allow the drop to occur. However, before the collision occurred the entropy was already changing simply because the ball and earth were approaching each other in what seems a less random arrangement.

Another argument could be that the entropy change is overall positive for the fall to occur, since the kinetic energy picked up by the falling object has increased entropy so that the entropy loss is overcome so that the total entropy nevertheless increases. This however is not consistent with the conservation of total energy. The potential energy of the system is computed as $m g h$ where $h$ is the distance between earth and the object,and $g$ is the gravitational field strength. This latent potential energy appears to drive the reaction forward to a lower potential energy with an associated increase in kinetic energy, all without transfer of heat, but with a decrease in entropy. Although entropy of the surroundings in chemical changes is typically due to heat gain, in a perfect vacuum there is no possibility of heat exchange and yet this system would still proceed with a change in entropy. The $m g h$ energy lost is exactly the same in magnitude as the kinetic energy $\left(1 / 2 m v^{2}\right)$ the object gains.

Finally, Isaac Newton's third law of Physics (1687) states that the object must pull on the earth as the earth pulls on the object with equal force. The force of the object of mass $m$ on the earth of mass $M$ would be $\left(m G /(r+h)^{2} M\right.$ where $G$ is the universal gravitation constant and $r$ is earth's radius. But this is identical to the force of the earth on the ball, $\left(M G /(r+h)^{2} m\right.$. Thus the total work done by the system is due to the earth and object actually moving toward each other. The force times distance work would be the same for each, but for a separation height $h$ of 1 


\section{Thermodynamics and Entropy in Natural and Artificial Systems}

meter, the distance moved by the object of nearly 1 meter is accompanied with a miniscule distance $d$ moved by the earth of about one trillionth of one femtometer. Here $M g d \approx m g(h-d)$ and since $d$ is negligible compared to $h, d \approx m h / M$. Formally, any change in enthalpy is given by

$$
\Delta H=\Delta E+\Delta(P V)
$$

where $E$ is the total internal energy $q+w$, heat plus work, and $P V$ is pressure volume work. In a vacuum here $P V$ and $q$ would be zero so $H=w$, and the entropy change of the surroundings should equal $w / T$. $w$ is equal in magnitude for the object. $\Delta S_{\text {total }}>0$ because the entire system contains a gravitational field with stored potential energy that is expended when the object and earth move toward each other. The total entropy change is the absolute value of the work done by both to disperse the energy, $2 w / T$. This total increase in entropy, while the two objects nevertheless join together, tells us a clue about the nature of gravity and is analogous to the release of a stretched rubber band or spring, as described in the next section.

\section{Entropy and Shape}

Gas expansion from a container into a larger volume in its surroundings represents a large increase in entropy of the gas. Often times an increased volume occupied by a system is an increase in randomness entropy. In other cases, increased entropy is associated with a rearranged shape of a system's volume. For example when a rubber band is stretched, the entropy is reduced even though the band lengthens to span a larger distance then when relaxed. To increase the entropy of the system merely release the stretched band to allow it to contract again. The reason the entropy then increases is because the stretched band has internal fibers that are aligned in a linear arrangement. Upon relaxation, the fibers disorient themselves again in a scattered pattern. Internal potential energy, imparted to the band upon stretching, is released to a minimum energy as the entropy increases. Further increases in entropy from contraction of the band shorter than natural rest length is blocked by an increased energy that would occur as for compressing a spring.

A mass forced onto a spring compresses the spring distance $x$ so that the spring contains internal potential energy (1/2) $k x^{2}$ where $k$ is the force constant of the spring in Newtons per meter. Release of the compressed spring increases entropy upon lengthening, similar to the rubber band when it shortens, by allowing the coils to randomize again while the internal energy dissipates to zero. Recoil expansion to lengthen the spring would also increase internal energy but the decrease in entropy again causes the spring to finally rest at its equilibrium length, neither contracted nor extended. Here energy is minimized and entropy is maximum. The reason entropy decreases if a spring is stretched is best seen by noticing that eventually stretching would cause the coils to form a linear arrangement. Entropy increases when a stretched spring or band is released, and so too for a mass falling in a gravitational field.

\section{Geochemistry and Climate}

The zeroth law of thermodynamics dictates that for any two objects in contact having differing temperatures, regardless of the total heat content of each, heat must exit the higher temperature object and enter into the cooler object, and heat transfer will continue until both objects attain the same temperature. As the earth orbits the sun to produce hemisphere warming during summer and hemisphere cooling during winter, atmospheric temperatures are higher and then lower than the temperature of polar pack ice. Pack ice of frozen seawater increases in area during the winter and thaws again in summer due to heat flow that is entropy driven. Heat from warmer surroundings warms and then melts frozen seawater at a melting point of $-2^{\circ} \mathrm{C}$ preferentially, compared to glaciers of frozen fresh water melting at $0^{\circ} \mathrm{C}$. In winter, heat from ocean water is absorbed by the surroundings causing re-freezing of the ice pack. In both cases total entropy increases.

Sea ice and glacier fresh water ice have comparable heats of melting $\left(334 \mathrm{~J} / \mathrm{g}^{\circ}\right)$ but the lower melting temperature of sea ice helps to protect melting of glaciers. The entropy gained by the ice melting must be larger than the entropy lost by the environmental surroundings for the melting to occur, because the heat entering the ice is 


\section{Thermodynamics and Entropy in Natural and Artificial Systems}

equal to the heat leaving the system, and it must be that $\Delta \mathrm{G}<0$. Each kilogram of pack ice that melts at $-2^{\circ} \mathrm{C}$ absorbs 334,000 J of heat energy from its surroundings with an entropy increase of $334,000 \mathrm{~J} /(271 \mathrm{~K})=1232$ $\mathrm{J} / \mathrm{K}$. For the sake of argument if all this heat were removed from an equal amount of seawater at $0^{\circ} \mathrm{C}$, then the entropy lost by this surroundings would be about $334,000 \mathrm{~J} /(/ 273)=1223 \mathrm{~J} / \mathrm{K}$, so the process under these conditions would be spontaneous toward melting, since $\Delta S_{\text {total }}=9 \mathrm{~J} / \mathrm{K}$. Glacial ice would not fully melt at this temperature since $\Delta S_{\text {total }}=0$, but melts at higher atmospheric temperatures.

At temperatures below $-2^{\circ} \mathrm{C}$, the reverse occurs and the ice freezes, where nevertheless again $\Delta S_{\text {total }}>0$. For any temperature above the melting point of fresh water icebergs, the total entropy increase for sea ice melting is greater per $\mathrm{kg}$ than for fresh water, so sea ice helps protect icebergs/glaciers from melting. For example, the entropy increase for sea ice at $271 \mathrm{~K}$ melting in the surrounding water at $1^{\circ} \mathrm{C}(274 \mathrm{~K})$ is $-334 \mathrm{~kJ} / 274 \mathrm{~K}$ $+334 \mathrm{~kJ} / 271 \mathrm{~K}=13.5 \mathrm{~J} / \mathrm{K}$. For fresh ice it is $-334 \mathrm{~J} / 274 \mathrm{~K}+334 \mathrm{~kJ} / 273 \mathrm{~K}=4.4 \mathrm{~J} / \mathrm{K}$. Warming melted water is a negligible contribution. Sea ice can remove far more heat from the surroundings than icebergs/glaciers can.

Note that the total entropy content, or the total energy content per $\mathrm{K}$, for the world's oceans is vastly larger than the total entropy content of a water area that freezes into pack ice when the surrounding water is below $-2^{\circ} \mathrm{C}$. This demonstrates the zeroth law, where heat nevertheless leaves the pack ice which is warmer than the surroundings, and enters into the water which is colder but contained more total entropy or heat. The entropy per mole or per gram, and thus the temperature, determines the direction of heat flow, not the amount of total heat the objects contain.

Melting and re-freezing of seawater does not alter sea levels because the frozen ice is seawater that expands in volume and with a lower density floats with $1 / 10^{\text {th }}$ of its volume above water while still having the same weight exerted on the ocean. Land-based glacier ice is fresh water which melts at $0^{\circ} \mathrm{C}$ and is thus less affected by these seasonal swings in temperature. Any melting of glaciers directly into the ocean, or indirect calving into icebergs that float with $1 / 8^{\text {th }}$ the volume above water that may melt later, adds additional water to the ocean.

Worldwide, many glaciers have been receding for several centuries, but at least one, the Brady Glacier, Glacier National Park, Alaska, has been growing. This indicates the complexity of heat transfer processes and climate cycles on earth. The extent of ice loss of the Grand Pacific Glacier, just 25 miles North of the Brady and first mapped by Capt. George Vancouver in 1794, has been rather steady. The 4,000 foot thick, 20 mile wide glacier receded from a length of 100 miles to 52 miles in length when examined by John Muir in 1879 . By 1916 the length was 35 miles (see Figure 8).

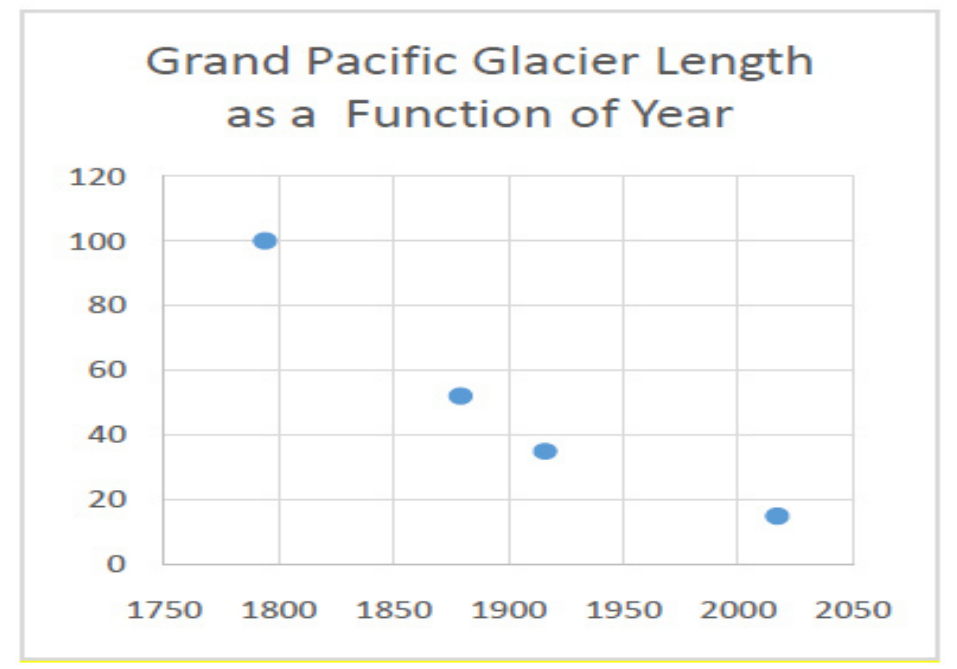

Fig8. The Grand Pacific Glacier has decreased from 100 miles in length to about 18 miles in length over a 223 year period. 


\section{Thermodynamics and Entropy in Natural and Artificial Systems}

Sunspots that become visible on a slow but regular basis cause significant increases in solar radiation intensity that modulate earth surface temperatures. Thus, a high frequency sinusoidal pattern of seasonal temperature variation is superposed on a low frequency sinusoidal variation caused by changes in the composition of the sun. An idealized chart is shown in Figure 9 for such a rhythmic variation in atmospheric temperatures for a planet as a function of time.

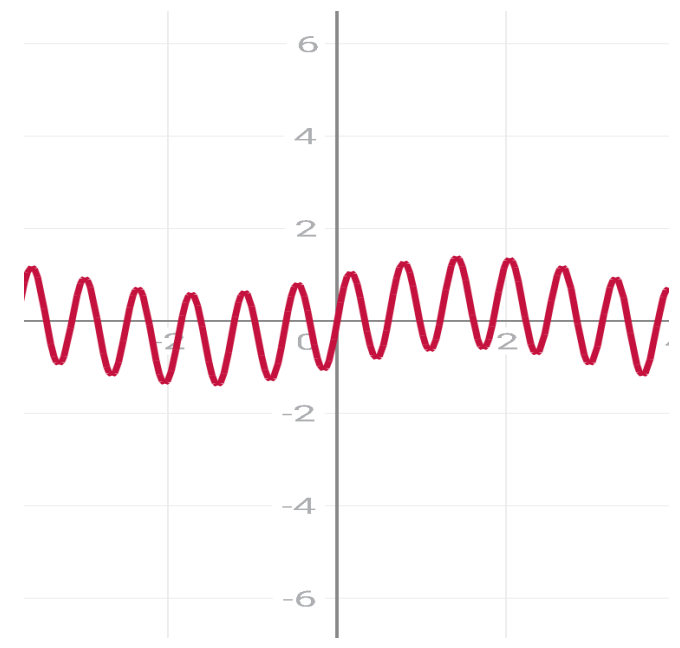

Fig9. Idealized plot of the temperature of a planet as a function of time that orbits the sun, which itself cycles heat-generating sunspots over longer periods. Multiple oscillations of high and low temperatures occur as the planet orbits between summer and winter yearly. Superposed fluctuations at much lower frequency occur due to two centuries-long cycles in the presence of sunspots. The axes coordinates are arbitrary.

Further, deep sea thermal vents, mineral hot springs and volcanic eruptions contain the massive quantity of heat that continuously reaches the earth's surface from intense deep thermal reactions. Although obviously these reactions have a high total entropy change and are very favorable, and the activation energy required to begin these unknown reactions has been achieved, the ongoing process is a long protracted reaction system since heat has been spewing to the surface for all observable time. Many ocean regions have been warming somewhat over the last decades and some have been cooling. Moreover, ever since the force causing Pangea to crack, tectonic plates have been spreading around the globe. This is due in part to the increased entropy associated with mixing or randomization of the plate materials. Relative tidal heights along shorelines is in part due to vertical shifts caused by plate collisions and erratic motion. The North American Plate continues Westward from the upthrust of material along the mid-Atlantic Ridge. This plate has collided with the Northward heading Pacific Plate that after the collision is now drifting Northwest. The collision may have cracked the Grand Canyon and formed the Sierra Nevada and other mountain ranges, while the Pacific Plate subducts continuously into the deep trench near Japan.

When the surroundings are warmer than any particular system such as a glacier, then heat flows into the system and the total entropy change $\Delta S_{\text {total }}$ is positive. And yet, when the temperature of the surroundings is less than the system, heat leaves the system into the surroundings, also with a positive total entropy change. This is well-known in Chemistry. Equal mass samples of warm water and frozen ice, in contact so that a given amount of heat transfers from one into the other, occurs spontaneously because the total entropy change is positive. The entropy gain from the colder ice is more than the entropy loss by the warmer water because the heat entered a colder system than the system from which it departed (1).

$$
\Delta S_{\text {total }}=\Delta S_{\text {system }}+\Delta S_{\text {surr }}=\Delta H / T_{\text {system }}+\Delta H / T_{\text {surroundings }}=-q / T_{\text {surroundings }}+q / T_{\text {system }} .
$$




\section{Thermodynamics and Entropy in Natural and Artificial Systems}

The cold ice has a larger entropy gain because its $T$ is lower. In the reverse situation, the heat gained by the surroundings is positive and again greater, being at the lower temperature. If the ice is at its melting point, the $\Delta q / T$ gain is the magnitude of the entropy change, but in that case the heat is converted into work, namely internal vibrations of the solid molecules, rather than causing the temperature to warm, since ice melting occurs at a fixed temperature. Entropy changes result from heat or any type of work changes in a system.

One particularly notable change is the Gulf of Mexico which has warmed a massive $10^{\circ} \mathrm{C}$ over the last several decades while the New Orleans area has sunk substantially below sea level. This may be due to the removal of an oil blanket under the Gulf that normally insulates and slows heat radiation to the surface, but this is a logical speculation where also a warmer equatorial Gulf Stream may be involved as well. Thus earth ice content, and atmospheric and water temperatures are affected by many processes.

Human activity appears to generate elevated levels of carbon dioxide in many measured locations in the atmosphere which is consistent with thick smog layers in large cities worldwide particularly during thermal inversions that impair health. Although this is argued to significantly increase the heat capacity of the atmosphere, the standard entropy content $S^{0}$ of oxygen gas $(205 \mathrm{~J} / \mathrm{K})$ is only slightly less than carbon dioxide $(214 \mathrm{~J} / \mathrm{K})(1)$ that it roughly replaces, where $S^{0}=q / T$ and is a measure of heat content per mole at standard temperature.

Of greater concern is that Henry's law, $s=k p$ where $s$ is gas solubility in solution and $p$ is the partial pressure of the gas above that solution, dictates that $\mathrm{CO}_{2}$ solubility in the ocean increases at higher partial pressures above it. Ocean acidification from dissolved $\mathrm{CO}_{2}$ forming carbonic acid,

$$
\mathrm{CO}_{2}+\mathrm{H}_{2} \mathrm{O} \rightarrow \mathrm{H}_{2} \mathrm{CO}_{3} \rightarrow \mathrm{H}^{+}+\mathrm{HCO}_{3}^{-} \text {， }
$$

can disintegrate calcium carbonate $\mathrm{CaCO}_{3}(\mathrm{~s})$ shells and coral reefs, where

$$
\mathrm{CaCO}_{3}(\mathrm{~s})+\mathrm{H}^{+} \rightarrow \mathrm{Ca}^{2+}+\mathrm{HCO}_{3}:
$$

Carbon dioxide levels measured above Mauna Loa, Hawaii have generally climbed by $24 \%$ since 1958 and passed $400 \mathrm{ppm}$ for the first time in 2013 (17). Ocean pH dropped from 8.25 to 8.14 in the last century which is a 30\% increase in acid content. This is entropy driven as well, since the elevated $\mathrm{CO}_{2}$ amount in the atmosphere is trapped by gravity so that the pressure of the gas is elevated, which is a reduced entropy state. The gas expands into the ocean accordingly.

Calcium ion and fluoride exist in the ocean at saturating levels where

$$
\left[\mathrm{Ca}^{2+}\right][\mathrm{F}]^{2}=[0.01 \mathrm{M}][.00005 \mathrm{M}]^{2}=2.5 \times 10^{-11}
$$

which is the $K_{\mathrm{sp}}$ or solubility product constant for the salt. This suggests that all fluoride in the ocean at $1 \mathrm{ppm}$ is from natural dissolved calcium fluoride. Therefore it is possible that calcium levels at $400 \mathrm{ppm}$ may eventually be reduced also by industrial fluoride emissions from waste water treated with fluosilicic acid, presumed to have dental benefit, but which is ineffective for this purpose and is a permanent bone accumulating toxic substance in man (18).

It must be emphasized that attempts to decrease carbon dioxide emissions are only useful if other pollutants are not created in its place, such as plutonium that is generated from nuclear fission reactors. Plutonium, with vast half-lives for its radioactive isotopes, cannot be neutralized. Adequate storage facilities are not known for the massive waste from these sites that have acute radiation toxicity at close range but most troublesome are radiation hazards lifetime after being inhaled. The plutonium content on earth naturally is nearly zero. One billion grams of earth typically contain only one billionth of one gram of plutonium. Complaints registered about these issues were unable to stop the construction of nuclear facilities that merely boil water for electricity. This has led predictably to deleterious outcomes, notable examples being Chernobyl, Russia, Fukushima, Japan, Three Mile Island, Pennsylvania, and the late San OnofreNuclear Generating Station (SONGS) in California with 


\section{Thermodynamics and Entropy in Natural and Artificial Systems}

vast tons of radioactive waste that cannot be neutralized or stored indefinitely near corrosive salt water. Dry storage in relatively thin steel casks planned at the SONGS site is no match for the fact that the entropy of the system will proceed forward. Surrounding waste with carbon rods and very thick steel casks, distant from salt air and urban sites, are a minimum for somewhat long-term containment.

The decrease in entropy associated with mining and collecting massive amounts of uranium into one facility has caused this irreversible toxic problem. Nuclear plants produce plutonium from the filthy fission reaction that also continuously releases low density radioactive gases from all such facilities with long term inhalation toxicity. These facilities should never have been constructed and now must be discontinued and decommissioned since storage remains unsolved. Nuclear missile tips are regularly replaced due to radiation degradation. While all such high level military waste is stored in underground caverns, fission reactor waste though is not allowed.

\section{Entropy Changes in Energetic Hurricanes}

The third law of thermodynamics defines a solid ordered crystalline substance at extremely low temperature as a state with so little molecular disorder as being zero entropy (realizing though that the material would still contain entropy since electrons are orbiting around jostling nuclei in the atoms of the crystal). On the opposite extreme are molecular systems that are completely mixed throughout a volume so that the energy the system contains is dispersed throughout.

The constant rotation of the earth produces a Coriolis effect, due to drag of the gaseous atmosphere and liquid oceans, so that ocean currents and wind currents prevail. When warm water warms air to low density that rises, the Coriolis swirling of gases can cause the occurrence of tornadoes, cyclones, typhoons, and hurricanes. Air molecules in some hurricanes can have tremendous organized kinetic energy with tangential velocities above170 miles per hour.

The hurricane system is actually a heat transfer device, where Joules of heat energy from the warm water transfers according to the zeroth law of thermodynamics upward through the hurricane to the very cold atmosphere above it. Some of the heat energy is converted into mechanical kinetic energy as well. The wind tangential velocity is no greater in magnitude than normal speeds that air molecules have while undergoing Brownian motion in the ambient atmosphere, at about 1,000 miles per hour. But the key reason the system has less entropy than ambient air is that the thermal energy input couples with the spinning earth and gravity to cause the molecules to have a directional angular velocity (counterclockwise in the Northern hemisphere), instead of random motion. The rotational energy $I w^{2}$ (where $I$ is the rotational inertia $\left(\mathrm{kg}-\mathrm{m}^{2}\right)$ and $w$ is the angular velocity in radians per second) in a large system becomes immense during late-year seasons when average wind speeds are slow and unable to disrupt the rotating system when it first begins to form.

So the developing hurricane behaves in some ways as dynamic equilibria do, with heat both entering and leaving the system, but while strengthening due to some of the heat being converted into mechanical energy of directed motion. The molecules turn inward because the low density warm air has lower pressure than surrounding atmospheric pressure which forces the flow inward. After hitting land, a different dynamic near-equilibrium occurs where heat flow upward from the ocean stops, heat dissipation occurs more laterally to the surroundings with molecular collisions and loss of directed motion until temperatures and energies of the gas match atmospheric.

High entropy systems usually result after molecular motion dissipates order and disperses mass energy to increase entropy as in Figure 2. What is not intuitive is that these fast-rotating hurricane systems are relatively low in entropy for the amount of heat and kinetic energy that they contain because the swirling molecules are an aggregate that is more ordered than ambient air. Entropy varies from the central eye, to the eye wall, to the outer edges, but is lowest in the eye wall where molecules travel in an ordered circular motion. Although warm ambient air contains more entropy than an equal quantity of air that is cooler under the same conditions of 
Thermodynamics and Entropy in Natural and Artificial Systems

temperature and pressure, the circularly swirling molecules are no longer in random motion but instead speed in an orderly path which represents a more ordered energy and thus a lower entropy.

The high wind speeds in the eye wall cause reduced pressure, because of Daniel Bernouilli's principle (1738), by $(1 / 2) \rho v^{2}(\rho$ is the density of agas or fluid and $v$ the velocity). High velocity substances decrease internal pressure, much like the mechanism by which airplane flight lift is caused by the lower pressure of the speeding molecules above the wing than below. Similarly, for water molecules moving rapidly in a thin section of a hose the pressure is reduced, compared to the same flow in a thick section of hose at low velocity. Thus internal pressures are lower, while internal temperatures are higher, inside the hurricane than the ambient surrounding atmosphere. Recent studies of hurricane Katrina indicated internal entropy progressively decreased as the hurricane developed with increasing wind speed (directed tangential to the rotating circle), and later at landfall, entropy increased as wind speed diminished (14), since heat input from the high-heat-capacity ocean ends there (Figure 10). Liquid ocean water can evaporate to transfer heat more efficiently than solid land.

\section{Time-Dependent Relative Thermodynamic Parameters in Hurricanes}

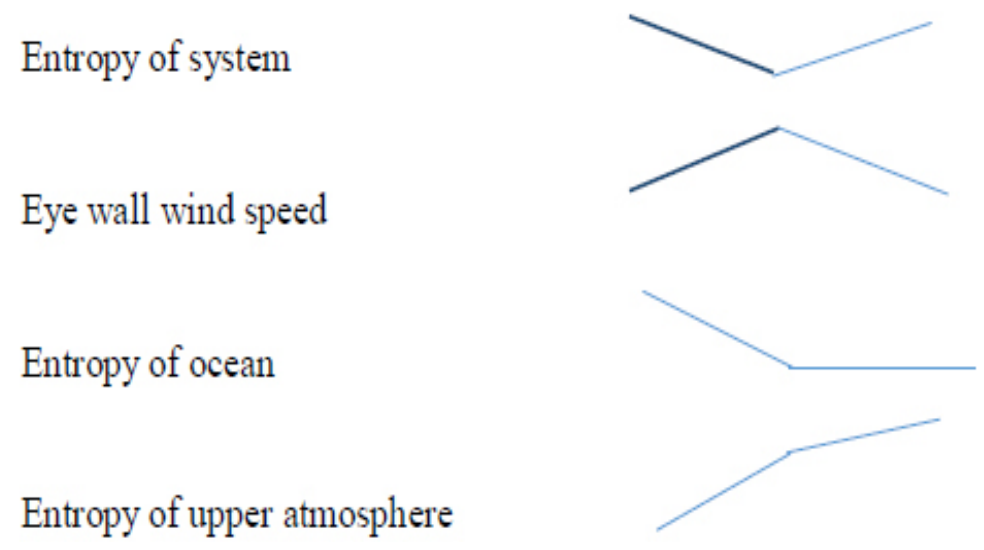

Fig10. Idealized relationship between internal entropy of a hurricane system, its external surroundings the ocean and upper atmosphere, and wind speed before (left half) and after landfall (right half). During buildup, heat from the warm ocean dissipates into the hurricane and orders molecules of air into circular motion since internal pressure of low density warm air is reduced and ambient surrounding pressure forces molecules to turn inward. Random Brownian molecular motion becomes circular motion having angular velocity which increases as warming continues. At landfall, the system dissipates since ocean heat input stops and collisions with ambient air impair circular motion and cause dispersion of energy so entropy rises. The total entropy change for both buildup and dissipation is positive since both processes occur naturally. The end result is much more heat is transferred from the ocean than the total heat gained by the atmosphere, so that the remainder performs work of devastation on land. $\Delta S_{\text {total }}=\Delta S_{\text {system }}+\Delta S_{\text {surr }}=\Delta S_{\text {system }}+\Delta S_{\text {ocean }}+\Delta S_{\text {atmosphere }}$. Over the open warm ocean during hurricane strengthening, $\Delta S_{\text {ocean }}<0, \Delta S_{\text {system }}<0$, and $\Delta S_{\text {atmosphere }}>0$. The large positive $\Delta S_{\text {atmosphere }}$ is due to the fact that the heat transfer to the upper atmosphere occurs into a region of very low temperature, typically $4^{\circ} \mathrm{C}$, and that $\Delta S=\Delta H / T$. At landfall, $\Delta S_{\text {ocean }}=0, \Delta S_{\text {system }}>>0$, and $\Delta S_{\text {atmosphere }}>0$. The high entropy gain at this stage is due to the massive conversion of molecules traveling as an ordered aggregate into a system with maximum mixing and normal Brownian motion again in ambient air. This entropy increase is due to randomness, as for the system shown in Figure 9 due to pressure, but here the increase is largely associated with loss of net directed angular momentum in the ordered motion that is known to occur. The heat loss to the lateral surroundings produces a heat gain that causes less entropy than that which occurred during buildup into the atmosphere above the hurricane system at the lower temperature. 


\section{Thermodynamics and Entropy in Natural and Artificial Systems}

Since both build-up and dissipation occur naturally, the total entropy must be positive in both cases. During build-up the $\Delta S_{\text {system }}$ decrease due to molecular ordering, with increasing wind tangential velocity, is matched by the $\Delta S_{\text {surr }}$ decrease from the ocean losing heat and the $\Delta S_{\text {total }}$ increase of the upper atmosphere gaining heat, so that the $\Delta S_{\text {total }}$ is positive for the spontaneous process. Recall that for a given amount of heat $q$ transferred, the entropy $q / T$ of the colder region is greater than the entropy lost from the warmer region supplying the heat. During breakdown, the $\Delta S_{\text {system }}$ gained by the hurricane, due to scattering and conversion of ordered tangential velocities to random motion as in ambient air, is coupled with heat loss to warm the environment and thus increase $\Delta S_{\text {surr }}$ so that the $\Delta S_{\text {total }}$ is again positive.

The net result is heat energy is transferred from the ocean to the atmosphere, but not in equal amounts. The difference between the total heat energy leaving the ocean and the lesser total heat energy entering the atmosphere is the energy available to do work, causing damage on land.

Collisions of these systems with land causes the orderly streaming gas to disperse, and the very large entropy increase of the system, with some loss of heat to the surroundings, leads to tremendous amounts of work and damage in the stricken region. Hurricane Katrina caused tremendous loss of life (up to 1,800 victims) and 108 billion dollars in damages in regions around New Orleans, Louisiana. In 2017 the one two three punch from hurricanes Harvey, Irma and Maria devastated Houston, Texas, much of Central Florida, Puerto Rico, and the U.S. Virgin Islands, the only portion of the entire United States that had been visited by Christopher Columbus in the $15^{\text {th }}$ Century.

The large negative free energy $\Delta G$ available to do work, causing devastation, is due to the large negative enthalpy change with heat loss from the system to its surroundings on land, plus the large positive entropy change with dispersion of energetic molecules from an ordered to a fully disordered state upon collision with stationary land structures. In this case the heat loss and entropy increase cooperate together. Heat radiation is enhanced from warm gas into the atmosphere since mixing with the surroundings increases exposure to more cooler air surfaces, much like the effect that stirring has to increase the rate that substances dissolve in solution. The amount of free energy for the overall process from ocean to land is $\Delta G=\Delta H-T \Delta S$ which is hugely negative and highly spontaneous since the large enthalpy loss by the system is negative and the entropy change from the overall dispersion of energy is positive.

Experimentally-generated tornado systems at the Reuben H. Fleet Space Science Center, Balboa Park, San Diego, California were found to quickly dissipate when a surprisingly small but non-rotating object is inserted into the system. To attempt to prevent devastation from hurricanes that are charted to be large and aimed at population centers, it would be prudent to examine the feasibility of dropping a large, but non-nuclear waste-producing, bomb into the eye of a hurricane while it develops in the remote open Atlantic Ocean. This should dissipate the system and prevent landfall of fully developed, high category hurricanes.

\section{Entropy-Controlled Versus Controlling Entropy}

Processes that involve energy dispersal are typically entropy driven in nature, some for the worse such as the dissipation of hurricanes on land, to many that are useful such as the wind-driven dispersal of pollen that fertilizes female parts of corn stalks. In other cases, system entropy decreases occur at the expense of entropy increases in the surroundings such as hurricane formation or the gathering of acorns by squirrels to store food. Notice that in the latter case the entropy is willfully controlled by the thoughts of the animal. Yes entropy can be controlled.

Willful entropy increases or decreases can be useful, such as the bomb-dispersed hurricane increase or the squirrel entropy decrease. Others would be the entropy decrease in water collected in a reservoir, or the entropy increase associated with water dispersal for drinking supplies for homes. In this case, again the potential energy developed in water collection freely dissipates spontaneously when released from the reservoir. Energy minimization couples with entropy maximization naturally in all such cases. 


\section{Thermodynamics and Entropy in Natural and Artificial Systems}

The original Joule experiment involved willfully pressurized gas with low entropy that contacted low pressure gas to demonstrate that entropy then drives the spontaneous reverse process of net gas movement to achieve full mixing. A simplified diagram based on his work is shown in Figure 2 for two containers with equal amounts of gas, $n$ moles. The gas in the left container has half the volume but twice the pressure so that $P V$ is constant, while the temperature is constant for both samples. Assume the system is blanketed from heat exchange with the surroundings. Opening a hole between the two chambers allows entropy to increase as the gas on the left disperses on a net basis throughout the entire container, and the gas on the right mixes in as well until static equilibrium is attained where the total pressure is now uniform, between the two original pressures. So

$$
\Delta S_{\text {total }}=\Delta S_{\text {system }}+\Delta S_{\text {surr }}=\Delta S_{\text {system }}>0 \text {. }
$$

Because both gases contained the same internal energy before mixing, where $E=n R T$ and $R$ is the ideal gas constant in liter-atmospheres per Kelvin-mole, and because there is no heat exchange with the surroundings, this form of entropy is exclusively due to randomization of the gas. One may also refer to it as energy dispersion because mass itself contains latent energy, and the energy concentration (energy per unit volume) is greater in the smaller volume before mixing to reach a uniform energy concentration throughout. $\Delta \mathrm{S}$ can be due to randomization of matter, internal energy changes, or both.

Entropy can be contained naturally indefinitely in many cases. Oil and ocean basins prevent the dispersion of these liquids. A forest fire is a system that increases its entropy by forming carbon dioxide gas from the burned organic matter, but if a lightning strike does not occur then neither does this entropy increase.

Transporting oil in a cargo ship or accumulating enriched uranium in a facility is artificial entropy management. The ship blocks the spread of the oil, but the infamous Valdez oil spill in the Pacific Northwest indicates how entropy must be taken seriously when it is attempted to be managed. The huge oil spill harmed wildlife and involved massive cleanup mitigation. The incorporation of oil into mussels and other fish species is well-known (15).

Enriching uranium is essentially irreversible, and accumulating it so that interatomic fission reactions begin is a facility with no off switch. The artificial process is an entropy driven mistake. The Hanford, Washington waste disposal site leaks are unable to be permanently contained, and waste drums at nuclear facilities that are stored in dry casks only temporarily contain the endlessly produced radioactive waste gases. Although it would be a massive undertaking, one method would be to dilute the enriched material with soil to former radiation levels when mined, and to refill the mine from which the material was first removed.

\section{Light}

Thermodynamic considerations in one example are difficult to reconcile. An isolated photon of energy traveling through free space must not violate thermodynamic truths, but the reason that this is so is unclear in terms of entropy considerations. Light photons are massless, infinitesimally small packets of electromagnetic energy that travel forward at fixed fast speed. Light photons travel in perpetuity over great distances, if not interrupted by absorbing, reflecting, scattering, diffracting, or refracting materials. The detection of photons on earth, that traveled successfully through deep space from stars thousands of light years distant, all arrive having the same speed cand particular frequency for energy where $E=h f$ and $h$ is Max Planck's (1900) constant, as when the photons first formed. Thus energy can be transported across great distances without energy loss (6).

Since this occurs, obviously the phenomenon does not violate thermodynamic truths. Although Newton's first law of Physics originally was derived for objects with mass, it appears that photons of massless electromagnetic energy follow this principle also, as an entity in motion that continues in motion when not interrupted by an entity that can change that motion. It must be that photon travel is spontaneous and thermodynamically favorable, so arguments extrapolated form thermodynamic truths, that there is no such entity as a perpetual motion machine or an engine with perfect efficiency without energy loss, do not appear to apply to light.

American Research Journal of Chemistry

Page 19 


\section{Thermodynamics and Entropy in Natural and Artificial Systems}

Further, there is no change in randomness entropy of the system as photons travel through empty space. But since the process is spontaneous, this suggests that there must be an entropy change in the surroundings that produces an overall positive total entropy. Here

$$
\Delta S_{\text {surr }}=\Delta H / T=(q+w+P V) / T=w / T
$$

since $P$ and $q=0$. Since $\Delta S_{\text {total }}$ must be positive for any spontaneous process, this implies that work $w$ is being done by the system on the surroundings through which the photon passes, but without loss of energy as the photon transports itself. Since light is energy and all light we now understand is produced from electron vibrations at temperatures $T>0$, the fact that this energy passes through points in space may be the equivalent of performing work on the surroundings. When light is absorbed by ocean water to produce heat, after traveling great depths, this is not due to friction since light has no mass. It is an electromagnetic interaction where water is a strong dielectric. Light passes through glass with a speed less than in air but returns to the faster speed upon re-entering the air because light has no mass and there is no friction. Light passing through deep space in a vacuum of course also would experience no friction or energy loss while propagating forward.

\section{Solar System Dynamics}

This returns us full circle back to consideration of mass. For an object in motion in deep space, the motion remains constant if uninterrupted by external forces, as stipulated by Isaac Newton's first law of Physics. Since mass contains latent energy (from $E=m c^{2}$ ), then this also represents a situation where energy is transported through space in perpetuity. Thus the first law of motion is consistent with thermodynamic truths since work may again be technically expended on the surroundings so that $\Delta S_{\text {total }}$ is positive to render the phenomenon spontaneous and favorable.

When planets orbit a star such as our sun, the increase in entropy of the surroundings due to transport of energy through space must also be involved. Here however the planets also turn inward because of the external gravitational force applied from the star, and from the planet on the star. In this way the entropy of the system, or planet plus star, does not increase on average since the planet is prevented from linear uninterrupted travel, thus preventing separation and escape into space and any increased randomness. The source of this negative entropy is not a heat term but is again the work achieved by the system where gravity causes a force times distance term causing an internal compression of the system.

This means that the overall process of orbiting by planets is entropy-neutral, where

$$
\Delta S_{\text {total }}=\Delta S_{\text {surr }}+\Delta S_{\text {system }}=0,
$$

and the system is in equilibrium. But why then does the orbiting process continue in perpetuity? This is because all systems in equilibrium still undergo dynamic processes. The saturated salt solution for example is in static equilibrium where also $\Delta S_{\text {total }}$ is 0 . Nevertheless, ions do dissolve into solution but at amounts equal to ions that precipitate from solution. Electrons that rapidly travel around nuclei in wave-like orbital patterns are in a static equilibrium state while Coulombic attraction and centripetal acceleration forces compete to cause translational kinetic energy and potential energy to continuously interchange. Similarly, planets that orbit stars are in an equilibrium state where on a net basis the planet neither escapes from the star nor collapses into the star, while continuously orbiting in perpetuity.

This system arrangement ensures that no energy is actually expended on a net basis and that in the absence of additional significant forces the system will remain in static equilibrium indefinitely, with the sum of potential and kinetic energy always a constant as planets oscillate in elliptic orbits. It has been found from NASA published observations, and from theory, that all planets contain twice as much kinetic energy as the negative potential energy required to remove the planet from its orbit (19). Like a rock initially thrown upward which is slowed, stopped, and accelerated downward over time, planets may be viewed as always falling toward the star while 


\section{Thermodynamics and Entropy in Natural and Artificial Systems}

orbiting. For half the orbit, the planet recedes and gains potential energy while decelerating and losing kinetic energy. For the other half, the planet approaches and loses potential energy while accelerating and gaining kinetic energy. But like diffusion of gas or liquid molecules in solution, there is no net transport of mass. In other words there is no net displacement of mass, like speeding objects traveling in circles that have no net velocity.

The total energy of an orbiting planetary system is constant at any position throughout the orbit. This is because the change in potential energy equals the magnitude of change in kinetic energy as the planet successively decreases and increases its distance from the sun. The total entropy is also constant so that $\Delta \mathrm{S}_{\text {total }}=0$ throughout the orbit as well. The entropy appears to increase while the planet recedes from the sun and decrease when approaching the sun. However, again the transport of mass energy through space involves a corresponding change in work so that the entropy remains constant throughout the orbit (Figure 11).

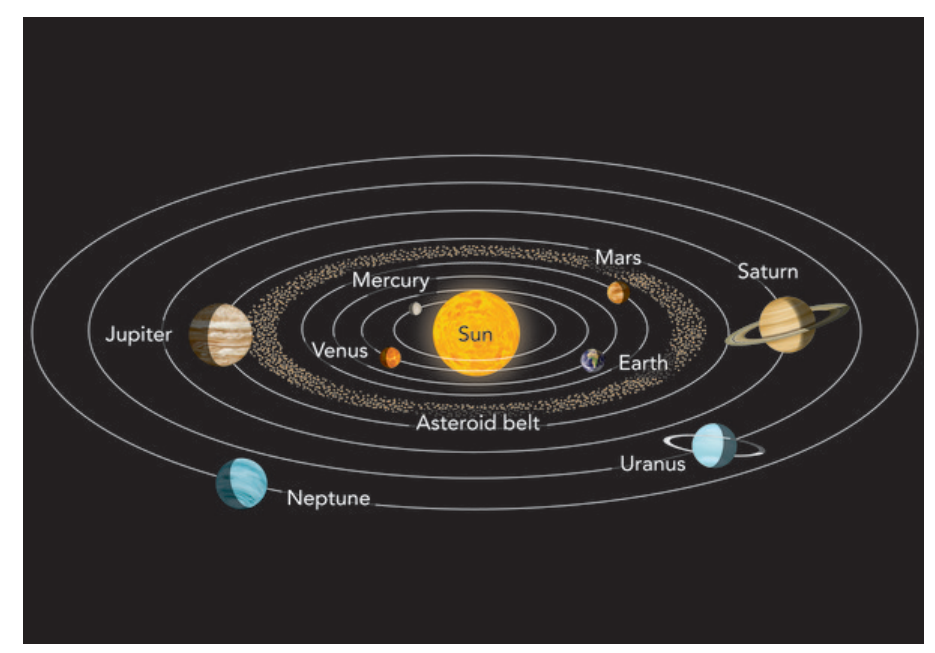

Fig11. Planets orbit the sun on the solar system ecliptic plane. The static equilibrium is nevertheless a dynamic process. Planets cannot escape to increase entropy but also cannot collapse into the sun to lose all potential energy. Each orbits with no net displacement or net velocity and thus do not exhibit net mass transfer in a given direction. So that $\Delta$ Stotal $=0$.

Similar to the thermodynamics of gases where expansion is a loss of internal energy and work by the system, when planets attempt to escape with increasing entropy due to randomness, the work term is positive. When gravity attempts to cause planets to fall, the entropy is negative but the work is negative so the total entropy remains constant. A photon or even objects with mass traveling in a vacuum of space with a linear velocity exhibits a positive total entropy change since energy is being transported from one place to another. But for an orbiting planet the total entropy is zero because there is no net transport of energy. Velocity is a vector quantity. Much like a spinning top, an orbiting planet has angular momentum but no net displacement and thus has a velocity of zero (Figure 11).

Being in a state of static equilibrium without energy loss other than decreased mass from the sun's fusion reactions, many interesting stunning relationships among the planets have been found. Mercury orbits about 1,000 times for each orbit of Pluto. The diameter of the moon is precisely $27.3 \%$ of the diameter of the earth, and 27.3 earth rotation days are required for the moon to complete each orbit (2). 27.3 days is also the time required for the equator of the sun to rotate on its own axis, the Carrington rotation (7). Thus the moon and sun share the same angular velocity of 0.230 radians per day. The diameter of the moon is the precise size to cause full solar eclipses of the earth at its position between sun and earth.

The very early conclusion of Isaac Newton, that the solar system was created to operate as a vast wound clock, is indeed insightful. But the solar system is even far more than that. An earlier study (8) presented how the moon 


\section{Thermodynamics and Entropy in Natural and Artificial Systems}

orbiting the earth indefinitely due to gravitational attraction generates tidal energy of the rotating earth's oceans indefinitely without actual energy loss. This is a profound result of the dynamism within a static equilibrium state and may result from the entropy associated with transport of mass energy through space that alternates rhythmically over the oceans that also rotate on earth indefinitely.

\section{The Universe and the First Law of Thermodynamics}

This analysis may be extended to galaxies in space and the entire material universe. A mole of a chemical substance contains 600 billion trillion $\left(6 \times 10^{23}\right)$ molecules. Approximately one mole of stars have been detected in the known universe. Ignoring the local burnout of stars and collisions of galaxies for the sake of argument, the entire universe of rotating galaxies would then be argued to exist approximately in a state of static equilibrium with each other, with many dynamic processes going on. Entropy changes that reflect internal energy dispersions in any planet are essentially isolated thermodynamically from each other.

Earlier arguments were discussed why the universe is not necessarily "expanding" (8). This may be also due to the balance between the drive to increase randomness entropy, with the drifting toward minimum energy driven by gravitational attractive forces of galaxies upon each other. For example nature abhors a vacuum, where evaporation is accelerated by evacuating a chamber. But atmospheres on planets and the materials in stars to not evaporate into the vacuum of space because gravitational forces oppose it. Thus entropy gains by galaxies if they were to expand from each other would be balanced by gravitational attraction between them (Figure 12).

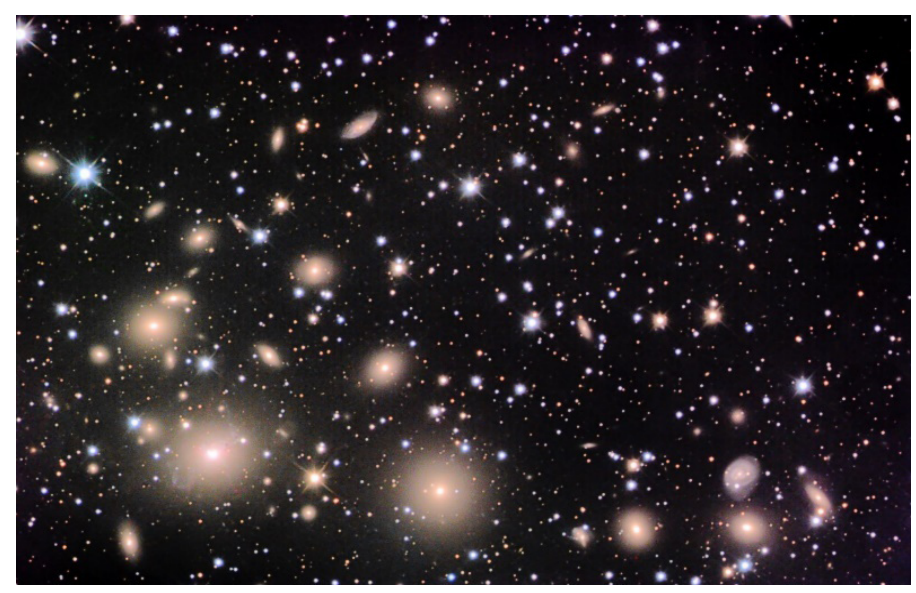

Fig12. Sample region of space with a vast number of galaxies. The entire universe contains an estimated one mole, or 600 billion trillion stars. Each galaxy is thermodynamically isolated from others so, ignoring energy dispersion that occurs in each and the formation or disappearance of individual stars over time, the universe may be thought of as a vast system that may be roughly in static equilibrium. The tendency for galaxies to separate to increase entropy is balanced by gravitational attraction where again each neither collapse into one mass nor escapes into deeper space.

The first law of thermodynamics, expressed as

$$
E=q+w,
$$

states that energy, including mass energy, in a system plus its surroundings is constant and cannot be either created to an increase or destroyed to a decreased total. In calculus differential form, $d E / d t=0$. This fundamental concept has infinite consequences. 


\section{Thermodynamics and Entropy in Natural and Artificial Systems}

First, the total energy present in the entire universe system of matter plus its infinite surroundings, as mass, heat, chemical, physical, light, electrical, magnetic forms, is now and has always been constant.

Second, since all the vast stars in solar systems now convert mass into light energy from $E_{\text {mass }}=m c^{2}$, matter has not always existed, and currently energy is being converted among various forms striving for energy dispersion and maximum entropy. The original universe system of mass thus was produced eons ago, from energy that existed eternally, where the entropy of the mass is low when formed, at the expense of energy expenditure elsewhere.

Third, the universe is well characterized as an ordered system where the earth is situated at a proper distance from the sun so that water and life can exist at moderate temperatures and has the right magnitude of gravity so that the atmosphere remains trapped with $20 \%$ oxygen at a density that can be breathed. The universe contains much order in subsystems such as galaxies with solar systems in ecliptic planes. The earth and other planets lie on a plane and were not splattered out of the sun since the sun is tilted with respect to the plane and at least one planet spins in a direction opposite the others. This ordering is inconsistent with an energy source randomly converting into mass.

Taken together, this is all consistent with the Newton description that the universe was created and is not the result of a random accident. The reduced entropy when the universe of matter was first formed would violate the second law if the surroundings were not also involved. Indeed, the reduced entropy of acorns assembled into a nest is not due to random self-assembly of the system into an aggregate that would violate the second law. It takes a squirrel in the surroundings to construct an ordered acorn storage assembly and an energetic God to first order the universe of matter to have all these characteristics and yet where $\Delta S_{\text {total }}>0$.

The constant energy of the entire universe of matter $(d E / d t=0)$ was originally, as now, not evenly distributed, and thus energy dispersion remains ongoing (where $d S / d t>0$ ). However, this does not mean that matter will eventually expand into even deeper space, because gravitational forces oppose that. Energy dispersal resulting from collisions of stars, or thermal diffusion of heat from inside the earth or other planets to the surface, or the nuclear fusion of stars slowing down, etc. are occurring within the universe without exchange from one subsystem to another. This entropy driven dispersion attempts to eventually achieve a minimum energy and maximum entropy for all subsystems, much like a gaseous atmosphere attempts to expand but is prevented by gravity from spewing into deep space.

Light produced from stars that is not interrupted which travels into deep space would represent loss of entropy from the system into the infinite surroundings. Here, and if this system were to drift with a linear velocity, the transport of energy through space would have $\Delta S_{\text {total }}>0$. In the distant future if energy dispersion were to ever become complete, in the absence of supernatural input, no further entropy change would occur, as in the Joule experiment, where

$$
d S_{\text {total }} / d t=d S_{\text {system }} / d t=d S_{\text {surroundings }} / d t=0 .
$$

Meanwhile, from the first law, $d E / d t=0$ since the universe has been in existence.

\section{Origins and change through time}

Physics texts commonly discuss cosmologic significance of physical findings, so here also a mention is warranted. The idea that the source of energy used to first form the material universe, that rendered the process 


\section{Thermodynamics and Entropy in Natural and Artificial Systems}

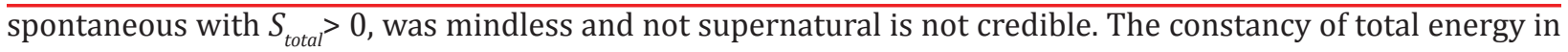
the universe means that energy has been eternal.

The alternative notion, that no source of energy outside the entire universe of matter exists, is also not tenable, since matter cannot be created from nothing. Mass energy can only be formed from pre-existing energy. From the first law, the source of energy is eternally constant but has been interconverted to form the material universe. Since time cannot be reversed, the material universe cannot be reconverted back to massless energy without additional energy expenditure, as though the forward reaction never took place. Indeed, time dilation in special relativity theory has been thoroughly disproven experimentally, theoretically, and mathematically (6). Just as total entropy increases for any directed natural process, absolute time proceeds only forward. Energy to mass conversion, as well as the first appearance of living organisms from inanimate matter, are not thermodynamically reversible processes.

After the first formation of mass from energy produced an ordered universe of matter, living organisms were formed, observed only on earth, that have undergone microevolution ever since due to genetic rearrangements, mutations, epigenetic factors, and natural selection observed in prospective experiments to produce a wide variety of subspecies within a particular genus. No evidence of macroevolution outside a genus has ever been observed. The opposing notion that the universe originated (from $E=m^{2}$ ) that eventually led to the first living things which on earth underwent macroevolution through natural selection and genetic modification due to mindless random processes has no visible evidence in prospective experiments with the scientific method, or support from thermodynamics.

\section{CONCLUSIONS}

The zeroth, first, second, and third laws of thermodynamics are presented in a useful way. Although it is usually argued that directed natural processes can only take place if the total entropy change associated with it is positive (2), this must be clarified. Equilibrium states, such as the saturated salt solution where ions dissolve and precipitate constantly, orbiting spatial bodies, where kinetic and potential energies continuously interchange, and electron oscillations in atomic orbitals are a few examples of processes that proceed indefinitely over time even though the total entropy remains constant. Positive total entropy changes occur for all spontaneous natural processes that strive toward equilibrium. This natural property can be willfully utilized for useful purposes, but requires consideration to protect from significant adverse risk.

\section{ACKNOWLEDGMENT}

Gratitude goes to many fine Christian women and men over the years: Andrew A. Benson (1917-2015), who discovered the $\mathrm{CO}_{2}$ fixation product in plant photosynthesis, worked until 97, and was a long-time mentor and friend; and my dad, Pearl Harbor survivor, for saying "stay in school." Thanks go to Palomar College students for interest, including Minjung Jo for reading the manuscript. Don Lovitt is thanked for comments on pack ice. Edwin Espinoza is appreciated for valuable editing and production work.

\section{DISCLAIMER}

The conclusions and clarifications in this manuscript are those of the author and do not necessarily reflect the views or mission of Palomar College. Please feel free to agree or to disagree with the contents. All California Community Colleges are supported in part by the U.S. Government and thus defend the U.S. Constitution including Amendment I, protect rights of free speech, do not discriminate against lawful religious or cultural beliefs, and 
Thermodynamics and Entropy in Natural and Artificial Systems

promote teaching and searching whole truths. California Education Code anti-dogmatism law prohibits either proselytizing or teaching information on origins as scientifically proven fact, rather than theory. Compiled during the 2017 Christmas season.

\section{REFERENCES}

1. McMurry, J. and Fay, R. Chemistry, 6 ${ }^{\text {th }}$ Edition, Prentice Hall, Upper Saddle River, NJ, 2012.

2. Giancoli, D., Physics, $6^{\text {th }}$ edition, Upper Saddle River, NH, 2005.

3. Moore, W.J. Physical Chemistry, $3^{\text {rd }}$ edition, Prentice Hall, Inc. Englewood Cliffs, NJ, 1962.

4. Gould, E.E. Scientific American, Jan 20, 2013.

5. Encyclopedia Brittanica at https://cdn.digitru.st/prod/1.5.1/redirect.html?redirect=\%2F\%2Fscience\%2Fph otosynthesis\%2FBasic-products-of-photosynthesis

6. Sauerheber, R. On the nature of light and relativity, Physics Essays27(1) 2014: 116-125.

7. Solar Rotation, Wikipedia at: https://en.wikipedia.org/wiki/Solar_rotation

8. Sauerheber, R. Gravity Contrasted with Light and Other Fields; Energetics and Solar System Dynamics, 2016, available at: http://jamesrobertdeal.org/wp-content/uploads/Sauerheber-Gravity-energy-solar-systemdynamics-8-26-16.pdf

9. Sauerheber, R, Temperature Effects on Conductivity in Seawater and Physiologic Saline, Chemical Science Journal, 2013 at: https://www.omicsonline.org/open-access/temperature-effects-on-conductivity-ofseawater-and-physiologic-salinemechanism-and-significance-2150-3494-1000109.php?aid=63301

10. Gordon, L. M. and Sauerheher, R.D. Calcium and Membrane Stability, The Role of Calcium in Biological Systems, Volume II, Anghileri, L and Anghileri, A., eds., CRC Press, Boca Raton, FL 1982: 3-16.

11. Hyslop, P.A., Kuhn,„C.E. and Sauerheber,,R.D. Insulin stimulation of glucose transport in isolated rat adipocytes. Functional evidence for insulin activation of intrinsic transporter activity within the plasma membrane Biochemical Journal 232, 1985:245.

12. Hyslop, P.A., Kuhn, C.E. and Sauerheber, R.D. Insulin stimulation of adipocyte membrane glucose transport. A graded biologic response insensitive to bilayer lipid disordering, Biochemical Pharmacology 36(14), 1987:2305-2310.

13. Sauerheber, R.D., Kuhn, C.E., Frigeri, L.G., Preisman, R.A., Dubuc, P., Peabody, H.D., and Hyslop, P.A., Noninsulin dependent diabetes mellitus in obese hyperglycemic mice in relation to feeding: comparison with the human obese diabetic condition, Current Therapeutic Research 44(4), 1988:612-618.

14. C. Liu, Y. Liu, Z. Luo, X. Lei, D. Wang and X. Zhou (2011). Studies of Hurricane Evolution Based on Modern Thermodynamics, Recent Hurricane Research - Climate, Dynamics, and Societal Impacts, Prof. Anthony Lupo (Ed.), ISBN: 978-953-307-238-8, InTech, At: http://cdn.intechopen.com/pdfs/15327/InTech-Studies_of_the_ hurricane_evolution_based_on_modern_thermodynamics.pdf

15. Lee, R.F., Sauerheber, R. D., Benson, A.A., Petroleum Hydrocarbons: Uptake and Discharge by the Marine Mussel Mytilus edulis Science 177 1972:344-346.

16. Sauerheber, R.D. (2012)Biology Introduction; a 21 ${ }^{\text {st }}$ Century Formulation, www. lulu.com ID: 4594529. 
Thermodynamics and Entropy in Natural and Artificial Systems

17. ttps://climate.nasa.gov/400ppmquotes/

18. Sauerheber, R.D. Physiologic Conditions affect the Toxicity of Industrial Fluoride (used in water fluoridation), Journal of Environmental and Public Health 439490, 2013 at:https://www.hindawi.com/journals/ jeph/2013/439490/

19. Sauerheber, R. Calculus Principles in Solar System Dynamics 2017, available athttp://jamesrobertdeal.org/ wp-content/uploads/Sauerheber-pdf

20. Sauerheber, R.D., Gordon, L.M., Zimmerman, T.S., Esgate, J.A., and VanderLaan, W.P. Effects of Calcium, Lanthanum, and Temperature on the Fluidity of Spin Labeled Human Platelets, Journal of Membrane Biology 52, 1980.

21. Putkey, J.A., Speilvogel, A.M., Sauerheber, R.D., Dunlap, C.S., and Norman, A.W. “Vitamin D-mediated Intestinal Calcium Transport Effects of Essential Fatty Acid Deficiency and Spin Label Studies of Enterocyte Membrane Lipid Fluidity" Biochimica et Biophysica Acta688(1)1982:177-190.

22. Big Frog Mountain Corporation, Chattanooga, TN http://www.bigfrogmountain.com/SunHoursPerDay.html

23. Earth Sun Relationships and Solar Energy http://www.cengage.com/resource_uploads/ downloads/0495555061_137179.pdf

24. Panchen, Z., et.al., Substantial variation in leaf senescence times among 1360 temperate woody species, Annals of Botanyfile:///C:/Users/USER/AppData/Local/Temp/PanchenLeafSenesce.pdf

Citation: Richard D. Sauerheber, "Thermodynamics and Entropy in Natural and Artificial Systems". American Research Journal of Chemistry, 2(1); pp:1-26.

Copyright @ 2018 Richard D. Sauerheber, This is an open access article distributed under the Creative Commons Attribution License, which permits unrestricted use, distribution, and reproduction in any medium, provided the original work is properly cited. 